\title{
Enhancement of Tunneling Magnetoresistance Through a Magnetic Barrier of Granular Fe-Pb-O System
}

\author{
Jen-Hwa Hsu, Ching-Ray Chang, and Yi-Hong Huang
}

\begin{abstract}
A large value of magnetoresistance ratio has been observed in granular $\mathrm{Fe}-\mathrm{Pb}-\mathrm{O}$ solids. The large MR value is associated with the appearance of magnetic $\mathrm{Pb}$ ferrite encapsulated on the surface of iron granules. Spin-dependent tunneling picture can qualitatively describe the enhancement of magnetoresistance ratio. Calculated results based on the spin filter effect of the $\mathrm{Pb}$-ferrite layer are reasonably in agreement with the experimental data of TMR enhancement in Fe-Pb-O solids.
\end{abstract}

Index Terms-Tunneling magnetoresistance, spin-dependent, granular film, spin polarization.

\section{INTRODUCTION}

$\mathbf{T}$ UNNELING magnetoresistance effect (TMR) draws much attention for its fascinating transport properties and industrial applications [1]. Usually the change of resistance with magnetic field in a tunneling junction is much higher than that in multilayers, and the power consumption of the junction is less but with higher sensitivity. Therefore, TMR is particularly utilized in nanostructured memory device [2] for the advantage of heat reduction, e.g., magnetic random access memory. In spin-polarized tunneling studies, polarization of the tunneling current is found to originate from the Zeeman splitting of the density of states of spin-up and spin-down conduction electrons in the ferromagnetic electrode. Since the TMR ratio is expected to be proportional to the product of the spin polarization of both ferromagnets [3], many efforts have been devoted to find ferromagnetic materials with large value of polarization. However, the spin polarization of natural ferromagnetic material at room temperature is at most 0.44 [4] and thus expected TMR ratio is limited to $32 \%$.

After the observation of significant magnetoresistance of a trilayer junction with an insulating spacer [5], some granular systems with insulating matrix also exhibit TMR effect [6]. Electron tunneling between ferromagnetic granules, the electron spin polarization within ferromagnetic granules as well as the relative orientation of the magnetization of different granules determines the value of TMR [7]. Usually a nonmagnetic oxide is the choice for the insulating matrix of the granular

Manuscript received January 20, 2000. This work was supported by National Science Council, ROC with Grant NSC88-2112-M002-009.

J.-H. Hsu and C.-R. Chang are with the Dept. of Phys., National Taiwan University, Taipei, Taiwan 106 (e-mail: \{jhhsu; crchang\}@phys.ntu.edu.tw).

Y.-H. Huang was with the Department of Phys., National Taiwan University, Taipei, Taiwan 106. He is now with Trace Storage Technology Co., ScienceBased Industrial Park., Hsinchu, Taiwan (e-mail: yhhuang@ tracetec.com.tw).

Publisher Item Identifier S 0018-9464(00)08759-8. system [6]. Recently some theoretical calculations suggest that a spin-dependent barrier can enhance the polarization and thus the TMR value [8], [9]. Also a granular $\mathrm{Fe}-\mathrm{Pb}-\mathrm{O}$ system has been reported to possess a large TMR value as long as a magnetic phase of $\mathrm{Pb}$ ferrite has been formed; however the reason for the significant enhancement of TMR is not well understood. In this work we study the possible mechanism which causes the enhancement of magnetoresistance ratio through the magnetic barrier in the granular $\mathrm{Fe}-\mathrm{Pb}-\mathrm{O}$ system. The observed enhancement of TMR in granular $\mathrm{Fe}-\mathrm{Pb}-\mathrm{O}$ films with the formation of magnetic $\mathrm{Pb}$ ferrite, at least a factor of ten, can be explained in the framework of spin-filter effect from a magnetic barrier.

\section{EXPERIMENT}

The $\mathrm{Fe}-\mathrm{Pb}-\mathrm{O}$ granular films were obtained from a new and unique method. The first step of preparation was to make metastable $\mathrm{Fe}-\mathrm{Pb}$ alloy films that were fabricated by magnetron rf sputtering with a composite target made from $\mathrm{Fe}$ and $\mathrm{Pb}$ powder. The substrate of kapton foil was cooled by LN2. Afterwards, films were annealed in the oven with flowing $\mathrm{Ar}$ gas for one hour. The oxidation also occurred in this process with residual oxygen in the oven. The detailed description of the preparation procedures has been reported elsewhere [10]. To compare with other $\mathrm{Fe}-\mathrm{Pb}-\mathrm{O}$ granular films, $\mathrm{Fe}(\mathrm{PbO})$ granular films were prepared with a composite target of $\mathrm{Fe}$ as well as $\mathrm{PbO}$ and by rf sputtering technique [11].

The film structure was determined from X-ray powder diffraction and the film morphology was investigated by transmission electron microscopy (TEM). A conventional four-probe method has been used to obtain the resisitivity of the granular film between $5 \mathrm{~K}$ and $300 \mathrm{~K}$. The magnetoresistance ratio, $[\rho(0)-\rho(H)] / \rho(0)$, was measured at room temperature with a maximum applied field of 0.9 Tesla. Under this field, the samples are not completely saturated.

\section{THEORY}

It has been suggested that a magnetic insulator between the ferromagnetic metals will enhance the spin polarization of tunneling electrons [8], [9]. For the ferromagnet-magnetic insulator-nonmagnetic insulator-ferromagnet (FM-MI-I-FM) junction, the ferromagnetic barrier acts as a spin-filter, and it selects the passing polarized electrons. Because of the presence of magnetic barrier, the attenuation lengths, $1 / \kappa_{\uparrow}$ and 


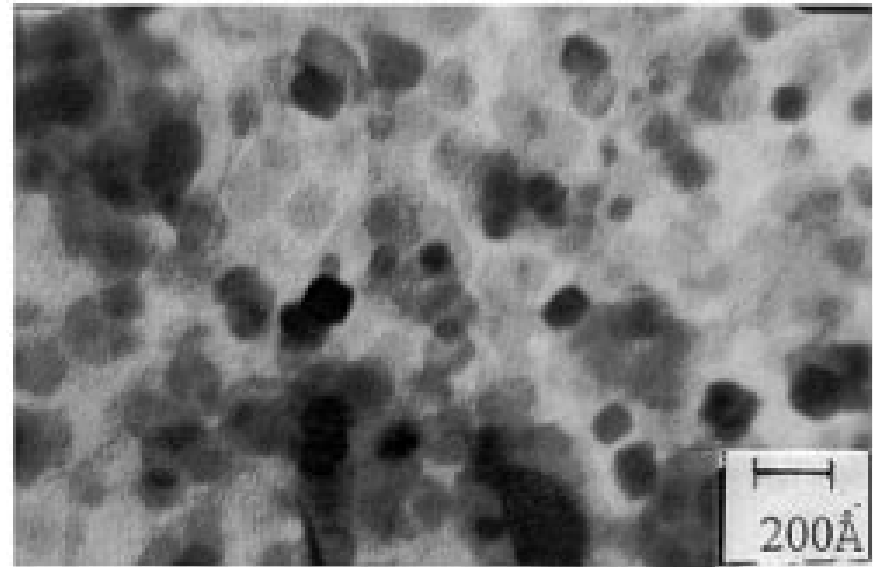

Fig. 1. TEM picture of Fe-Pb-O granular film.

$1 / \kappa_{\downarrow}$, are quite different for spin-up and spin-down electrons, respectively. Therefore the effective spin polarization through the spin-filter barrier, $P_{\text {eff }}$, is [8]

$$
P_{\text {eff }}=\frac{k_{\uparrow^{\prime}}-k_{\downarrow^{\prime}} \frac{\kappa_{\uparrow}^{2}-k_{\uparrow^{\prime}}^{2}}{\kappa_{\downarrow}^{2}+k_{\downarrow^{\prime}}^{2}}}{k_{\uparrow^{\prime}}+k_{\downarrow^{\prime}} \frac{\kappa_{\uparrow}^{2}+k_{\uparrow^{\prime}}^{2}}{\kappa_{\downarrow}^{2}+k_{\downarrow^{\prime}}^{2}}} .
$$

where $k_{\uparrow^{\prime}}, k_{\downarrow^{\prime}}$ are for the right ferromagnetic materials. For the nonmagnetic barrier, $P_{\text {eff }}$ reduces to Slonczewski's results [4]. For $U_{\uparrow}>U_{\downarrow}, \Delta \rho / \rho$ can be expressed as

$$
\frac{\Delta \rho}{\rho}=\frac{2 P_{\text {eff }}}{1+P_{\text {eff }}} .
$$

It should be noted that the TMR ratio of a conventional spin tunneling junction is proportional to the square of the spin polarization [3] while TMR ratio of a spin-filter junction is only proportional to the spin polarization. Since spin polarization is always less than 1, the spin-filter junction has a much larger TMR ratio than the conventional spin-tunneling junction. Enhancement of the TMR ratio from the spin-filter effect with a ferromagnetic barrier is quite notable, especially when the spin polarization is small. With a FM-MI-I-MI-FM structure, TMR ratio will approach $100 \%$ from the spin-filter effect.

\section{RESULTS AND DisCUSSIONS}

For films annealed at $300^{\circ} \mathrm{C}$, the resistivity of the films decreases with decreasing temperature, typical behavior of a metal. However, when the annealing temperature is above the melting temperature of $\mathrm{Pb}$, which is $327^{\circ} \mathrm{C}$, the resistivity of the films exhibits a tunneling behavior. The temperature dependence of the resistivity follows the tunneling behavior, i.e., $\ln \rho \propto T^{-1 / 2}$. The TEM picture of the granular films annealed at $400^{\circ} \mathrm{C}$ is shown in Fig. 1. High resolution TEM suggests some granules are core/shell structure where the dark core region is surrounded with other materials of light shell region (Fig. 1). The size of the granules is about 100-200 $\AA$, the area of shell is estimated to be about $40 \%$ of the granules and the radius of Fe-core is about 70-150 $\AA$. The distance between granules is roughly $50 \AA$. In Fig. 2 the X-ray diffraction pattern points out that in the shell a nonmetallic magnetic phase has formed, which is identified to be $\mathrm{PbO} \cdot 3 \mathrm{Fe}_{2} \mathrm{O}_{3}$ and the granules are embedded in the $\mathrm{PbO}$ matrix.

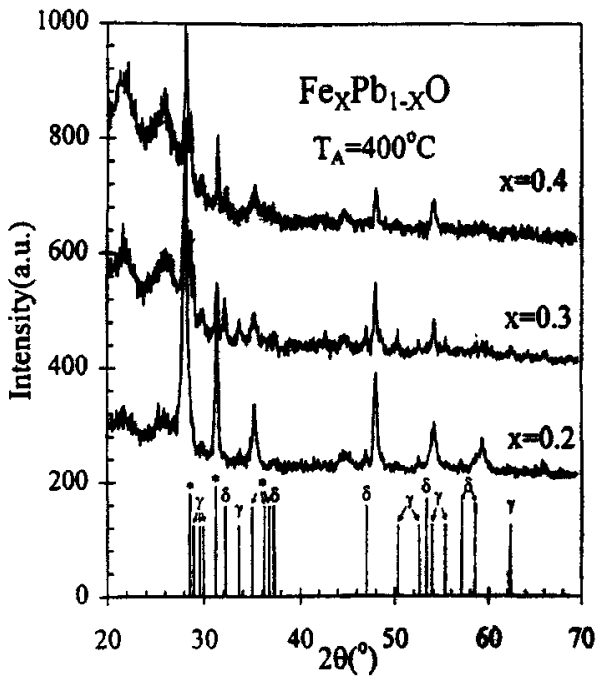

Fig. 2. X-ray diffraction patterns for annealed $\mathrm{Fe}-\mathrm{Pb}-\mathrm{O}$ granular films. (*): $\mathrm{PbO} \cdot 3 \mathrm{Fe}_{2} \mathrm{O}_{3} ;(\gamma): \mathrm{PbO} \cdot 2 \mathrm{Fe}_{2} \mathrm{O}_{3} ;$ and $(\delta): \mathrm{PbO} \cdot 1 / 2 \mathrm{Fe}_{2} \mathrm{O}_{3}$.

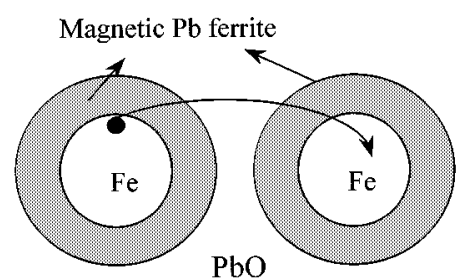

(a)

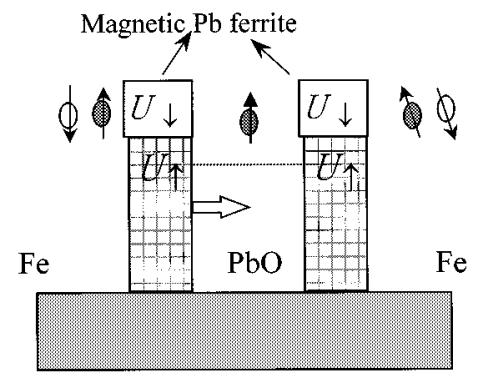

(b)

Fig. 3. (a) A schematic diagram of the electron tunneling between granules within $\mathrm{PbO}$ matrix. The granules are core/shell structure: Fe core surrounded with the magnetic $\mathrm{Pb}$ ferrite shell. (b) Mechanism of the enhancement of polarization through a spin-dependent potential of magnetic barrier. Spin-up electrons and spin-down electrons experience different spin-dependent potential barrier $U_{\uparrow}$ and $U_{\downarrow}$ respectively. The dash line is the barrier height for non magnetic $\mathrm{PbO}$ insulator. Only one kind of spin electrons emerges from the ferrite shells if the spin-filter effect is strong enough.

The TMR value is about $10 \%$ under $H=0.9$ Tesla at room temperature with $x=0.4$ for films annealed at $400^{\circ} \mathrm{C}$, It drops to $4.5 \%$ for films with $x=0.2$. It is apparent that the TMR ratio is reduced with decreasing amount of $\mathrm{PbO} \cdot 3 \mathrm{Fe}_{2} \mathrm{O}_{3}$. Furthermore, the TMR ratio becomes less than $1 \%$ for $\mathrm{Fe}(\mathrm{PbO})$ films where iron granules without $\mathrm{PbO} \cdot 3 \mathrm{Fe}_{2} \mathrm{O}_{3}$ ferrite shell are embedded in $\mathrm{PbO}$ matrix [11].

Since both TEM and X-ray data indicate the possibility that $\mathrm{Fe}$ cores are encapsulated within the $\mathrm{Pb}$ ferrite shell, the possible schematic structure of the $\mathrm{Fe}$ core/ $\mathrm{Pb}$ ferrite shell granules embedded in the $\mathrm{PbO}$ matrix is shown in Fig. 3(a). With the magnetic barrier, spin-up electron and spin-down 
electrons experience different tunneling height of the barrier [Fig. 3(b)] and their attenuation lengths are quite different. From (1), the spin polarization of the electron from the Fe core will be greatly enhanced as the electrons emerge from the $\mathrm{Pb}$ ferrite shell. If the tunneling mechanism of the electrons is described as in Fig. 3, the TMR ratio should approach $100 \%$ with the FM-MI-I-MI-FM structure. However, some of the Fe granules are not surrounded with the $\mathrm{Pb}$-ferrite shell and most of the electrons are still not tunneling through the magnetic shells on both sides. The experimental results should be described with FM-MI-I-FM structure as in (2) and the TMR ratio only depends on $P_{\text {eff }}$, instead of square of $P_{\text {eff }}$ in the Julliere model. No matter what kind of metals next to the ferromagnetic barrier, the electrons emerging from the ferromagnetic barrier have about the same polarization. Instead of the predicted $32 \%$ TMR ratio of the Fe-I-Fe junction from Julliere's model [3], we expect a $61 \%$ TMR ratio in Fe-MI-I-Fe spin-filter structure from (2). Therefore, the ultimate enhancement of TMR ratio is $61 \%$ for Fe granules with 0.44 polarization. However, the predicated TMR ratio of $61 \%$ is much larger than the observed $10 \%$ in granular $\mathrm{Fe}-\mathrm{Pb}-\mathrm{O}$ film. The main reason for this discrepancy is that the encapsulation of most Fe cores by magnetic $\mathrm{Pb}$ ferrite shell is not complete. Other possibilities are the reduction of effective polarization from the barrier height and thickness [4] and the spin-flip during the tunneling [12]. Comparing with Julliere model [3] and (2), the magnetic barrier enhances the TMR value to a factor of the inverse of effective polarization. Since the effective polarization usually is around the order of magnitude of 0.1 [4], it is easy to understand that the TMR value of $\mathrm{Fe}$ granules encapsulated with the magnetic $\mathrm{Pb}$ ferrite will be $10 \%$ if the TMR value of $\mathrm{Fe}(\mathrm{PbO})$ granular films is $1 \%$ as observed in the experiment.

We have studied $\mathrm{Fe}-\mathrm{Pb}-\mathrm{O}$ granular solids and found that an order of magnitude enhancement of TMR is observed with the formation of magnetic $\mathrm{Pb}$ ferrite on the surface of $\mathrm{Fe}$ granules. The enhancement of TMR may possibly result from the spin-filter effect through a magnetic barrier. We also found that it is reasonable for TMR to increase a factor of ten with a spinfilter barrier. Further research on the spin-filter tunneling junction is necessary for possible huge TMR ratio and potential applications in memory device.

\section{REFERENCES}

[1] G. A. Prinz, "Spin-polarized transport," Phys. Today, vol. 48, no. 4, pp. $58-63,1995$

[2] J. L. Simonds, "Magnetoelectronics today and tomorrow," Phys. Today, vol. 48, no. 4, pp. 26-32, 1995.

[3] M. Julliere, "Tunneling between ferromagnetic films," Phys. Lett., vol. 4A, pp. 225-226, 1975.

[4] J. C. Slonczewski, "Conductance and exchange coupling of two ferromagnets separated by a tunneling barrier," Phys. Rev., vol. B39, pp. 6995-7002, 1989.

[5] J. S. Moodera, L. R. Kinder, T. M. Wong, and R. Meservey, "Large magnetoresistance at room temperature in ferromagnetic thin film tunnel junctions," Phys. Rev. Lett., vol. 74, pp. 3273-3276, 1995. T. Miyazaki, T. Yaoi, and S. Ishio, "Large magnetoresistance effect in $82 \mathrm{Ni}-\mathrm{Fe} / \mathrm{Al}-$ Al2O3/Co magnetic tunneling junction," J. Magn. Magn. Mater, vol. 98, pp. L7-L9, 1991.

[6] H. Fujimori, S. Mitani, and S. Ohnuma, "Tunnel-type GMR in metal-nonmetal granular alloy thin films," Mater. Sci. Eng., vol. B31, pp. 219-223, 1995. Y. H. Huang, J.-H. Hsu, and J. W. Chen, "Thickness dependence of tunneling magneto-resistance effect in granular Fe-Al2O3 films," IEEE Trans. Magn., vol. 33, pp. 3356-3358, Sept. 1997.

[7] L. Xing, Y. C. Chang, M. B. Salomon, D. F. Frenkel, and J. Shi, "Magnetotransport properties of magnetic granular solids: the role of unfilled d band," Phys. Rev., vol. B48, pp. 6728-6731, Sept. 1993.

[8] C.-R. Chang and S. P. Chen, "Spin polarized tunneling through a ferromagnetic barrier," Chin. J. Phys., vol. 36, pp. 85-90, 1998.

[9] - "Enhancement of tunneling magnetoresistance through a magnetic barrier," J. Magn. Magn. Mater, vol. 61, pp. 209-212, Jan. 2000.

[10] Y.-H. Huang, J.-H. Hsu, J. W. Chen, and C.-R. Chang, "Granular Fe-Pb-O films with large tunneling magnetoresistance," Appl. Phys. Lett., vol. 72, pp. 2171-2173, Apr. 1998.

[11] J.-H. Hsu and Y.-H. Huang, "Tunneling magnetoresistance effect in $\mathrm{Fe}-\mathrm{Pb}-\mathrm{O}$ and $\mathrm{Fe}-\mathrm{PbO}$ granular films: A comparison," J. Magn. Magn. Mater, vol. 203, pp. 94-96, 1999.

[12] S. F. Zhang and P. Levy, "Magnetoresistance of magnetic tunneling junction in the presence of a nonmagnetic layer," Phys. Rev. Lett., vol. 81 pp. $5660-5663,1998$ 